\title{
Non-Abelian field strength bilocal correlator at finite temperature in the model of dilute instanton gas
}

\author{
N.O. Agasian * S.M. Fedorov ${ }^{\dagger}$ \\ June 5, 2004 \\ Institute of Theoretical and Experimental Physics, \\ 117218, Moscow, B.Cheremushkinskaya 25, Russia
}

\begin{abstract}
Bilocal correlator in gluodynamics is evaluated at finite temperature in the framework of instanton gas model. It is demonstrated that vacuum correlation length decreases with a growing temperature. Obtained results are compared with lattice data for the bilocal correlator at finite temperature. Density of instantons and possible structures of nonperturbative vacuum are discussed.
\end{abstract}

\section{Introduction}

Nonperturbative fields play important role in the vacuum of QCD and gluodynamics, and determine many properties of the theory, in particular, they are responsible for the confinement, spontaneous chiral symmetry breaking (SCSB), and as a result, for the formation of observed hadron spectrum. A well developed and successful in the description of many properties of QCD model is the instanton liquid model, suggested by Shuryak [1] and Diakonov and Petrov [2]. The main nonperturbative fields in this picture are well separated instantons and antiinstantons, their interaction being not very strong (thus the name "liquid"). Instanton density is approximately $1 \mathrm{fm}^{-4}$. This model solves a number of problems in QCD, in particular it naturally leads to SCSB, explains the $\eta^{\prime}$ meson mass. However, there is a number of problems of fundamental importance: it remains unclear, how to make such instanton-antiinstanton ensemble stable (infrared inflation of instantons), and confinement cannot be explained in the instanton liquid model.

However, apart from quasiclassical instantons there are other nonperturbative fields in the vacuum, which allow to solve "infrared problem" of instantons. Nonperturbative vacuum of QCD can be parametrized by gauge invariant vacuum averages of gluon field strength tensor - vacuum correlators [3]. This allows to successfully describe many phenomena in QCD (see the review papers [4 [5]). Bilocal correlator is sufficient for qualitative description of many phenomena (stochastic vacuum model). Moreover, there are indications that corrections due to higher correlators are small

*agasian@heron.itep.ru

†fedorov@heron.itep.ru 
and in some cases amount to several percent [4. Nonzero string tension naturally appears in this approach and can be expressed through vacuum average of chromoelectric field [3]

$$
\sigma=\frac{g^{2}}{2} \int d^{2} x\left\langle\operatorname{Tr}\left(E_{i}(x) \Phi(x, 0) E_{i}(0) \Phi^{\dagger}(x, 0)\right)\right\rangle,
$$

where $E_{i}=F_{4 i}$ is chromoelectric field and $F_{\mu \nu}=F_{\mu \nu}^{a} t^{a}$ is the field strength tensor of gluon field. $\Phi(x, 0)$ is the parallel transporter along the straight line, connecting points $x$ and 0 . Integration in (11) goes in space-time plane $(i, 4)$.

The problem of infrared inflation of instantons is also naturally solved in the stochastic vacuum model. Investigation of influence of nonperturbative quantum fluctuations on instantons was started in papers 6, 7]. Later it was shown that standard perturbation theory changes in the nonperturbative stochastic vacuum, and contribution of large instantons to physical quantities becomes finite [8]. Next, direct interaction of instanton with nonperturbative vacuum fields was shown [9] to lead to stabilization in instanton size, the average size of instantons being of the order of vacuum correlation length. Distribution of instantons over sizes was found to be in good agreement with lattice data, with a maximum at $\rho_{c} \simeq 0.25 \div 0.3 \mathrm{fm}$.

An important property of vacuum is the scaling of string tensions between sources in different representations of color group, which is observed in the lattice calculations 10. It was shown in 11] that instanton contribution to the force between heavy quarks in different representations violates Casimir scaling, and this leads to a constraint on instanton density.

In the present paper we study instantons' contribution to the bilocal correlator at finite temperature $^{1}$. We demonstrate that correlation length of bilocal correlator, evaluated on calorons (calorons are instanton-like configurations at finite temperature) in the lowest order in caloron density has significant dependence on temperature. At the same time it is found in the lattice calculations that within errors correlation length for chromomagnetic fields does not depend on the temperature in the whole region between zero and critical temperature $T_{c}[14$.

Temperature dependencies of the chromomagnetic bilocal correlator and of the spatial string tension, $\sigma_{s}(T)$, were found analytically in the paper [15. It was shown there that chromomagnetic condensate slowly grows with increasing temperature up to $2 T_{c}$, while correlation length $\lambda_{m}(T)$ does not depend on $T$ in this temperature region. At high temperature, $T>2 T_{c}$, condensate grows with $T$ as $\left\langle H^{2}\right\rangle_{T} \propto g^{8}(T) T^{4}$ and magnetic correlation length decreases as $\lambda_{m}(T) \propto 1 /\left(g^{2}(T) T\right)$. Obtained analytical expression for the $\sigma_{s}(T)$ agrees well with lattice results for all temperatures [16]. There exists a simple physical explanation of the independence of correlation length on the temperature at $T \leq T_{c}$. Inverse correlation length $M=1 / \lambda_{m}$ is a mass of lightest gluelump $1^{+-}$. Gluelumps 17] are not physical objects, however they play an important role in the nonperturbative QCD, because their masses determine field correlators, and in particular, string tension at $T=0$ is related to gluelumps. Their masses were calculated analytically in the framework of QCD sum rules [18, QCD string model [19] and were determined numerically in various lattice calculations 20. Summing up all those results, correlation length equals $\lambda_{m} \approx 0.15 \mathrm{fm}$, which corresponds to $M \approx 1.5 \mathrm{GeV}$. Taking into account that phase transition temperature is much less than gluelump mass $T_{c} \ll M$, it is physically clear that temperature dependence of gluelump mass, and therefore of the correlation length, should be very weak in the low temperature region $T<T_{c} \simeq 300 \mathrm{MeV}$, which is confirmed by analytical [15] and lattice [14 calculations. It should be mentioned, that magnetic correlation length does not change it's behavior at the phase transition

\footnotetext{
${ }^{1}$ Bilocal correlator in the instanton gas at zero temperature was evaluated in 12 [3].
} 
point. It is well known, that order parameter for the deconfinement phase transition is vacuum average of Polyakov loop, and therefore it is Polyakov loops correlator $\left\langle L(\vec{x}) L^{\dagger}(0)\right\rangle$ that significantly changes it's behavior at temperature $T=T_{c}$.

\section{Chromoelectric and chromomagnetic correlators}

At finite temperature euclidian $\mathrm{O}(4)$ space-time symmetry is broken to space $\mathrm{O}(3)$ symmetry, and bilocal correlator is described by independent chromomagnetic and chromoelectric correlation functions, which are expressed through four independent functions $D^{\mathrm{E}}\left(x^{2}\right), D_{1}^{\mathrm{E}}\left(x^{2}\right), D^{\mathrm{B}}\left(x^{2}\right) D_{1}^{\mathrm{B}}\left(x^{2}\right)$ :

$$
\begin{aligned}
& g^{2}\left\langle\operatorname{Tr}\left(E_{i}(x) \Phi(x, y) E_{j}(y) \Phi^{\dagger}(x, y)\right)\right\rangle=\delta_{i j}\left(D^{\mathrm{E}}+D_{1}^{\mathrm{E}}+z_{4}^{2} \frac{\partial D_{1}^{\mathrm{E}}}{\partial z^{2}}\right)+z_{i} z_{j} \frac{\partial D_{1}^{\mathrm{E}}}{\partial z^{2}} \\
& g^{2}\left\langle\operatorname{Tr}\left(B_{i}(x) \Phi(x, y) B_{j}(y) \Phi^{\dagger}(x, y)\right)\right\rangle=\delta_{i j}\left(D^{\mathrm{B}}+D_{1}^{\mathrm{B}}+\vec{z}^{2} \frac{\partial D_{1}^{\mathrm{B}}}{\partial z^{2}}\right)-z_{i} z_{j} \frac{\partial D_{1}^{\mathrm{B}}}{\partial z^{2}},
\end{aligned}
$$

where $B_{i}=\frac{1}{2} \varepsilon_{i j k} F_{j k}$ is the chromomagnetic field, $E_{i}=F_{4 i}-$ chromoelectric field and $F_{\mu \nu}=F_{\mu \nu}^{a} t^{a}$ is the field strength tensor.

$$
\Phi(x, y)=\operatorname{Pexp}\left(i g z^{\mu} \int_{0}^{1} d s A_{\mu}(y+s z)\right)
$$

is the parallel transporter along the straight line, connecting points $x$ and $y ; z=x-y$. These phase factors $\Phi$ are introduced to ensure gauge invariance of correlators. Obviously $D_{1}^{\mathrm{E}}=D_{1}^{\mathrm{B}}=0$, $D^{\mathrm{E}}=D^{\mathrm{B}}$ for the selfdual field (see [13]). Thus it is sufficient to consider chromomagnetic correlator only.

The very fact that for instantons $D^{\mathrm{E}}=D^{\mathrm{B}}$ implies that instantons cannot be the only nonperturbative fields in the vacuum of gluodynamics, because it is known from lattice calculations that chromoelectric and chromomagnetic condensates have different dependences on temperature. In particular, at the deconfinement phase transition temperature chromoelectric condensate abruptly vanishes, while chromomagnetic condensate stays intact [14].

\section{Calorons' contribution to the chromomagnetic correlator}

Instanton field with the center at the origin in the singular gauge is given by

$$
\begin{aligned}
& A_{\mu}^{a(\mathrm{inst})}(x)=-\frac{1}{g} \bar{\eta}_{\mu \nu}^{a} \partial_{\nu} \ln \Pi^{(\mathrm{inst})}(x), \\
& \Pi^{(\mathrm{inst})}(x)=1+\frac{\rho^{2}}{x^{2}}=1+\frac{\rho^{2}}{r^{2}+\tau^{2}},
\end{aligned}
$$

where $x=(\vec{r}, \tau)$. This expression is easily generalized to the case of finite temperature, and caloron has a well known form [21]:

$$
\begin{aligned}
& A_{\mu}^{a}(x)=-\frac{1}{g} \bar{\eta}_{\mu \nu}^{a} \partial_{\nu} \ln \Pi(x), \\
& \Pi(x)=1+\sum_{n=-\infty}^{\infty} \frac{\rho^{2}}{r^{2}+(\tau-n \beta)^{2}}=1+\frac{\pi \rho^{2}}{\beta r} \frac{\operatorname{sh}(2 \pi r / \beta)}{\operatorname{ch}(2 \pi r / \beta)-\cos (2 \pi \tau / \beta)},
\end{aligned}
$$


where $\beta=1 / T$ is the inverse temperature. Caloron field is given by

$$
\begin{aligned}
& F_{\mu \nu}^{a}=\partial_{\mu} A_{\nu}^{a}-\partial_{\nu} A_{\mu}^{a}+g \varepsilon^{a b c} A_{\mu}^{b} A_{\nu}^{c}, \\
& g B_{i}^{a}=-\delta_{a i} g_{1}(x)+\varepsilon_{a i j} \frac{x_{j}}{r} g_{2}(x)+\frac{x_{a} x_{i}}{r^{2}} g_{3}(x), \\
& E_{i}^{a}=B_{i}^{a}, \\
& g_{1}(x)=\frac{1}{r} \frac{1}{\Pi} \frac{\partial \Pi}{\partial r}+\frac{1}{\Pi} \frac{\partial^{2} \Pi}{\partial \tau^{2}}-\frac{1}{\Pi^{2}}\left(\frac{\partial \Pi}{\partial \tau}\right)^{2}+\frac{1}{\Pi^{2}}\left(\frac{\partial \Pi}{\partial r}\right)^{2}, \\
& g_{2}(x)=-\frac{2}{\Pi^{2}} \frac{\partial \Pi}{\partial r} \frac{\partial \Pi}{\partial \tau}+\frac{1}{\Pi} \frac{\partial^{2} \Pi}{\partial r \partial \tau}, \\
& g_{3}(x)=\frac{2}{\Pi^{2}}\left(\frac{\partial \Pi}{\partial r}\right)^{2}-\frac{1}{\Pi} \frac{\partial^{2} \Pi}{\partial r^{2}}+\frac{1}{r} \frac{1}{\Pi} \frac{\partial \Pi}{\partial r} .
\end{aligned}
$$

Owing to the selfduality of caloron field $F_{\mu \nu}=\tilde{F}_{\mu \nu}$ chromoelectric field is equal to chromomagnetic one.

Next step is the evaluation of the parallel transporter on caloron:

$$
\begin{aligned}
& \Phi(x, y)=\operatorname{Pexp}\left(i g z^{\mu} \int_{0}^{1} d s A_{\mu}(y+s z)\right)=\operatorname{Pexp}\left(-i z^{\mu} \bar{\eta}_{\mu \nu}^{a} t^{a} \int_{0}^{1} d s \partial_{\nu} \ln \Pi(y+s z)\right)= \\
& \quad=\operatorname{Pexp}\left(-i t^{a} \int_{0}^{1} d s\left[\bar{\eta}_{i j}^{a} z_{i} \partial_{j} \ln \Pi+\bar{\eta}_{i 4}^{a} z_{i} \partial_{4} \ln \Pi+\bar{\eta}_{4 j}^{a} z_{4} \partial_{j} \ln \Pi\right]\right) \\
& z=x-y
\end{aligned}
$$

For comparison with lattice results we will consider the case $z_{4}=0$. Then

$$
\Phi(x, y)=\operatorname{Pexp}\left(-i t^{a} \int_{0}^{1} d s\left[\bar{\eta}_{i j}^{a} z_{i} \partial_{j} \ln \Pi+\bar{\eta}_{i 4}^{a} z_{i} \partial_{4} \ln \Pi\right]\right) .
$$

In general one has to evaluate path ordered integral. However there are special cases, when the exponent commutes at different values of $s$. Obviously, these cases are $\tau=0, \tau=\beta / 2$ and $\tau=\beta$, since $\partial_{4} \ln \Pi(r, \tau=0 ; \beta / 2 ; \beta)=0$. Another example is the single instanton field, since $\partial_{4} \ln \Pi^{(\mathrm{inst})}=\tau(1 / r)\left(\partial \ln \Pi^{(\mathrm{inst})} / \partial r\right)$.

To evaluate the contribution of caloron gas to the two-point correlator in the lowest order in density one has to average single-caloron contribution over it's position, or, which is the same, to average over $y$ at a given $z=x-y$.

Averaging over $y$ comes to the integral $\int d^{4} y=\int d^{3} \vec{y} \int_{0}^{\beta} d y_{4}$. Averaging over three-dimensional vector $\vec{y}$ does not make any problems, but the integral over $y_{4}$ is not tractable, since correlator can be numerically calculated only when $\tau=0, \beta / 2, \beta$. Nevertheless it will be seen from comparison with the single-instanton case that at low temperatures calculations with $\tau=0$ are sufficient, and at high temperatures correlation lengths for $\tau=0$ and $\tau=\beta / 2$ cases coincide, which makes averaging trivial. 


\section{Numerical results}

Let us use the equation

$$
g^{2}\left\langle\operatorname{Tr}\left(B_{i}(x) \Phi(x, y) B_{j}(y) \Phi^{\dagger}(x, y)\right)\right\rangle_{\vec{y}}=\delta_{i j}\left(d+d_{1}+\vec{z}^{2} \frac{\partial d_{1}}{\partial z^{2}}\right)-z_{i} z_{j} \frac{\partial d_{1}}{\partial z^{2}}
$$

at $z_{4}=0$ to define the functions $d$ and $d_{1}$, which depend on $z^{2}$ and $y_{4} \equiv \tau: d=d\left(\tau, z^{2}\right) ; d_{1}=$ $d_{1}\left(\tau, z^{2}\right)$. Averaging over $y_{4}$ yields

$$
\begin{aligned}
& \int_{0}^{\beta} d \tau d\left(\tau, z^{2}\right)=D^{\mathrm{B}}\left(z^{2}\right) \\
& \int_{0}^{\beta} d \tau d_{1}\left(\tau, z^{2}\right)=D_{1}^{\mathrm{B}}\left(z^{2}\right)=0 \\
& D^{\mathrm{B}}\left(z^{2}=0\right)=\frac{4 \pi^{2}}{3}
\end{aligned}
$$

Last equality follows from the relation $g^{2} \int d^{3} \vec{y} \int_{0}^{\beta} d y_{4}\left(F_{\mu \nu}^{a}\right)^{2}=32 \pi^{2}$ (the field is selfdual, and this integral is proportional to the topological charge $\left.\sim \int d^{4} y F \tilde{F}\right)$.

Let us define correlation length $\lambda_{\tau}$ as the coefficient in the exponent of the best fit to the function $d\left(\tau, z^{2}\right)$ :

$$
d\left(\tau, z^{2}\right) \simeq e^{-|z| / \lambda_{\tau}}
$$

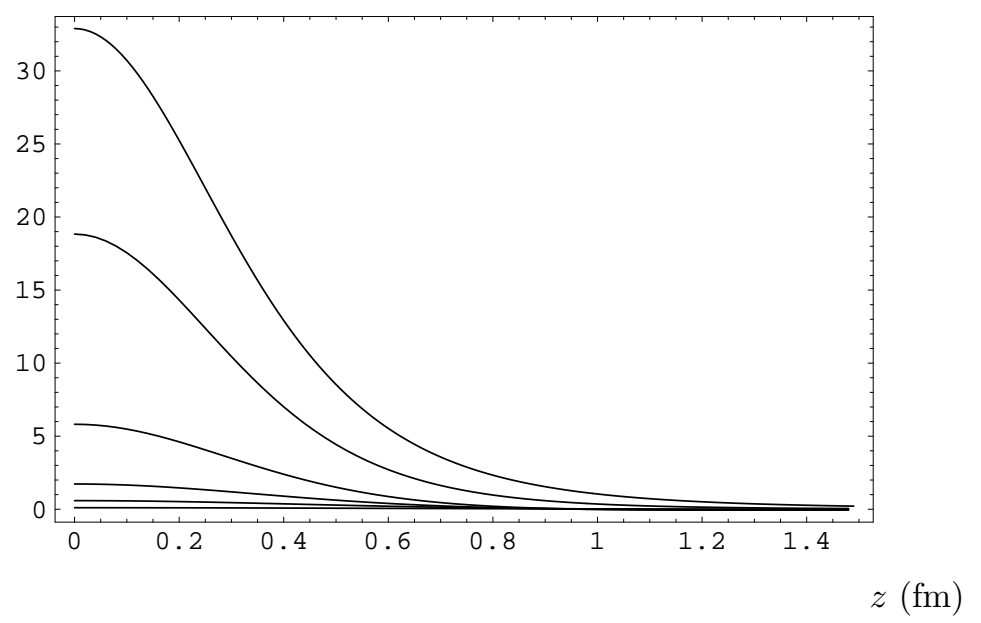

Figure 1: $\quad d^{\text {(inst) }}\left(\tau, z^{2}\right)$ at $\tau=(0,0.15,0.3,0.45,0.6) \mathrm{fm} ; \rho=0.3 \mathrm{fm}$. Upper line corresponds to $\tau=0$.

As discussed above, we will evaluate $d\left(\tau=0, z^{2}\right)$ and $d\left(\tau=\beta / 2, z^{2}\right)$ only. However, this information is sufficient to determine the correlation length of the function $D^{\mathrm{B}}\left(z^{2}\right)$. Indeed, by averaging over $\tau$ one can find that normalized to 1 functions $d\left(\tau=0, z^{2}\right) / d\left(\tau=0, z^{2}=0\right)$ and 


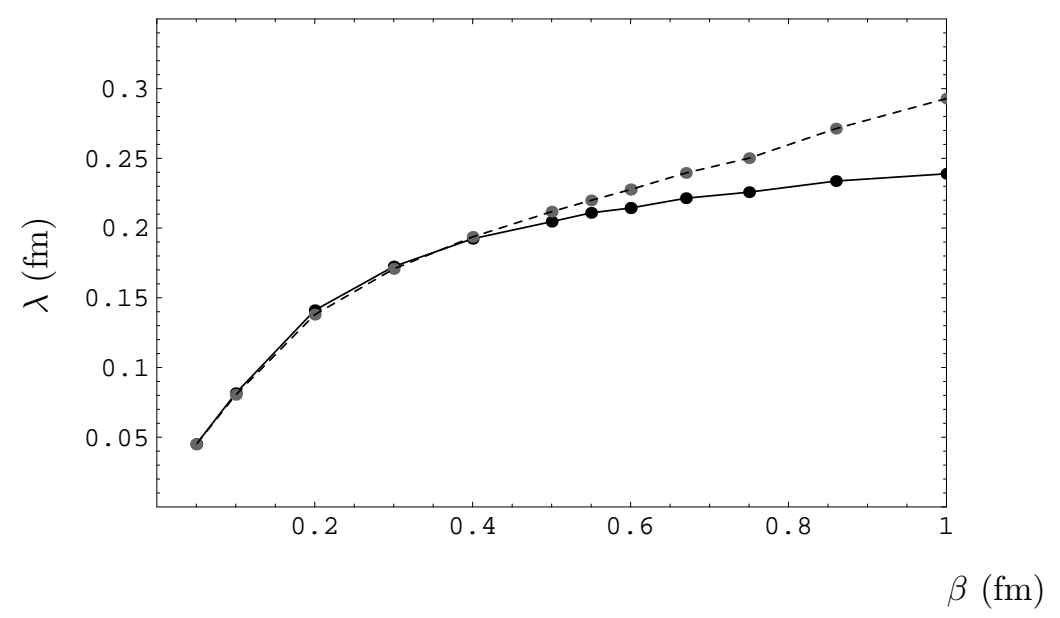

Figure 2: Correlation length of functions $d\left(\tau=0, z^{2}\right)$ and $d\left(\tau=\beta / 2, z^{2}\right): \lambda_{\tau=0}(\beta)$ (solid line) and $\lambda_{\tau=\beta / 2}(\beta)$ (dashed line); $\rho=0.3 \mathrm{fm}$.

$D^{\mathrm{B}}\left(z^{2}\right) / D^{\mathrm{B}}\left(z^{2}=0\right)$ practically coincide. The fact is that instanton is a well localized field configuration, and it's field decreases very fast when moving away from it's center. This is illustrated in the Fig. 1. It shows function $d^{\text {(inst) }}\left(\tau, z^{2}\right)$, calculated for the single instanton configuration for several values of $\tau$. Profile of the function $D^{\mathrm{B}}\left(z^{2}\right)$ is determined by the function $d\left(\tau=0, z^{2}\right)$. The same is true for caloron when $\beta \gg \rho$, because in this case instantons forming caloron are well separated. At higher temperatures, when $\beta \leq 2 \rho$ the functions $d\left(\tau=0, z^{2}\right)$ and $d\left(\tau=\beta / 2, z^{2}\right)$ have almost the same correlation length (see Fig. 2), which makes averaging over $\tau$ trivial (it only affects the amplitude of the functions, which can be found from the condition $\left.D^{\mathrm{B}}\left(z^{2}=0\right)=\frac{4 \pi^{2}}{3}\right)$.

Taking into account all aforesaid, we can restrict our analysis by the function $d\left(\tau=0, z^{2}\right)$ only. It's correlation length is close to the correlation length of function $D^{\mathrm{B}}\left(z^{2}\right)$ for all values of $\beta$.

Chromomagnetic correlator for $\tau=0, \beta / 2, \beta$ takes the form

$$
\begin{aligned}
& g^{2}\left\langle\operatorname{Tr}\left(B_{i}(x) \Phi(x, y) B_{j}(y) \Phi^{\dagger}(x, y)\right)\right\rangle_{\vec{y}}=g^{2} \int d^{3} \vec{y}\left(B_{i}^{a}(x) U^{a b}(x, y) B_{j}^{b}(y)\right), \\
& U^{a b}(x, y)=\operatorname{Tr}\left(t_{a} \Phi(x, y) t_{b} \Phi^{\dagger}(x, y)\right)=\frac{1}{2} \delta^{a b} \cos (2 \phi)-\frac{1}{2} \varepsilon^{a b c} n_{c} \sin (2 \phi)+n_{a} n_{b} \sin ^{2}(\phi), \\
& n_{a} \phi=\frac{1}{2} \bar{\eta}_{i j}^{a} z_{i} y_{j} \int_{0}^{1} d s\left(\frac{1}{r} \frac{\partial \ln \Pi(y+s z)}{\partial r}\right)
\end{aligned}
$$

Correlator defined in (12) is presented in Fig. 3 for several values of $\beta$. Corresponding correlation

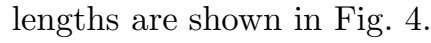

\section{Discussion}

Bilocal correlator at finite temperature has been studied on lattice in the paper [14], and it was found that bilocal chromomagnetic correlator practically does not depend on temperature in the 


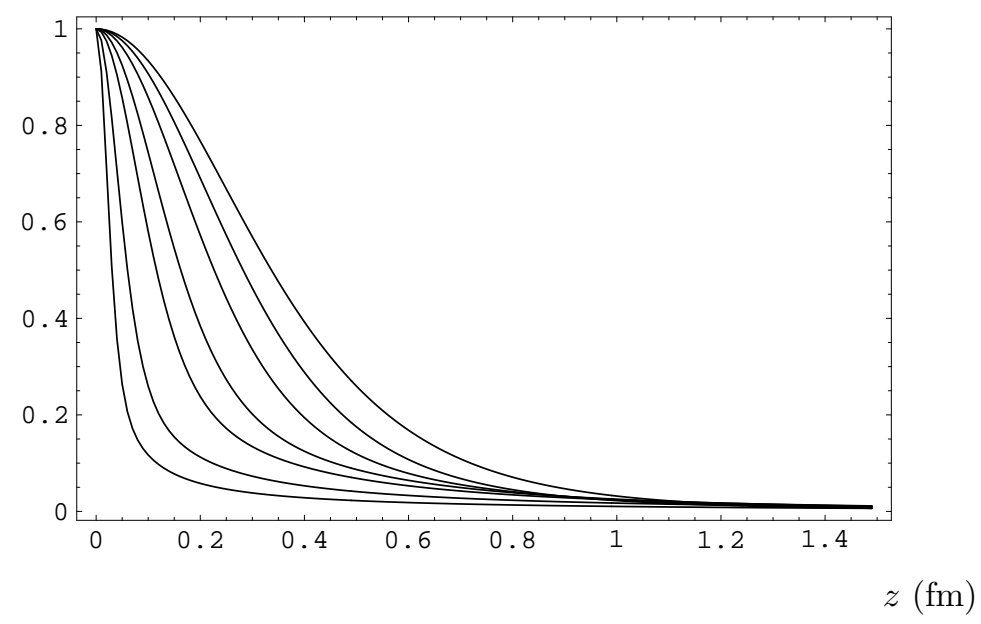

Figure 3: $\quad d\left(\tau=0, z^{2}\right) / d\left(\tau=0, z^{2}=0\right)$ for $\beta=(0.05,0.1,0.2,0.3,0.5,0.86, \infty) \mathrm{fm}^{-1} ; \rho=0.3 \mathrm{fm}$. The largest correlation length (upper line) corresponds to zero temperature $(\beta=\infty)$, i.e. to instantons' contribution.

whole region from zero to $T_{c}$ (this dependence is also very weak above critical temperature). At the same time, as it is seen from Fig. 4 the correlation length in the dilute instanton gas changes by approximately $20 \%$ when temperature changes from zero to $300 \mathrm{MeV}$. Thus, one comes to the conclusion that instanton gas cannot be a correct description of the real vacuum of gluodynamics. There are several possible explanations of these lattice data.

1) Instanton density is much less than $1 \mathrm{fm}^{-4}$, and the main contribution to the correlator comes from other nonperturbative fields, which are responsible for the confinement.

2) Instanton density is high enough to make next terms in density expansion change the result qualitatively, leading to the independent of temperature correlation length.

3) Instanton ensemble is rearranged at nonzero temperature, instantons and antiinstantons form molecules, and the contribution of these instanton-antiinstanton molecules to bilocal correlator is significantly different from that of instanton gas. This binding may be connected to the deconfinement phase transition. For example, this is the case for 3-d adjoint Higgs model, where it was found that in the deconfined phase instantons and antiinstantons are bound into molecules [22]. Formation of instanton-antiinstanton molecules in QCD vacuum and it's connection to the chiral symmetry restoration phase transition is discussed in review 23.

4) It should be noted, that in the last years a different scenario of the caloron vacuum in the confined phase $\left(T<T_{c}\right)$ is discussed. There exist field configurations with nontrivial holonomy, the so called KvBLL solution 24]. This scenario 25] suggests that calorons may be composites of dyons and antidyons. Lattice calculations, giving numerical evidence for the existence of these field configurations for the cases of $S U(2)$ and $S U(3)$ groups, are presented in papers [26] 27]. Clearly, in the case of this scenario correlation length in the nonperturbative vacuum has a more complicated temperature dependence, than in the case of caloron gas studied in this paper.

In principle, one can consider one possibility more, when instanton size distribution depends on temperature in such a way, that the resulting correlation length does not depend on $T$. However, 


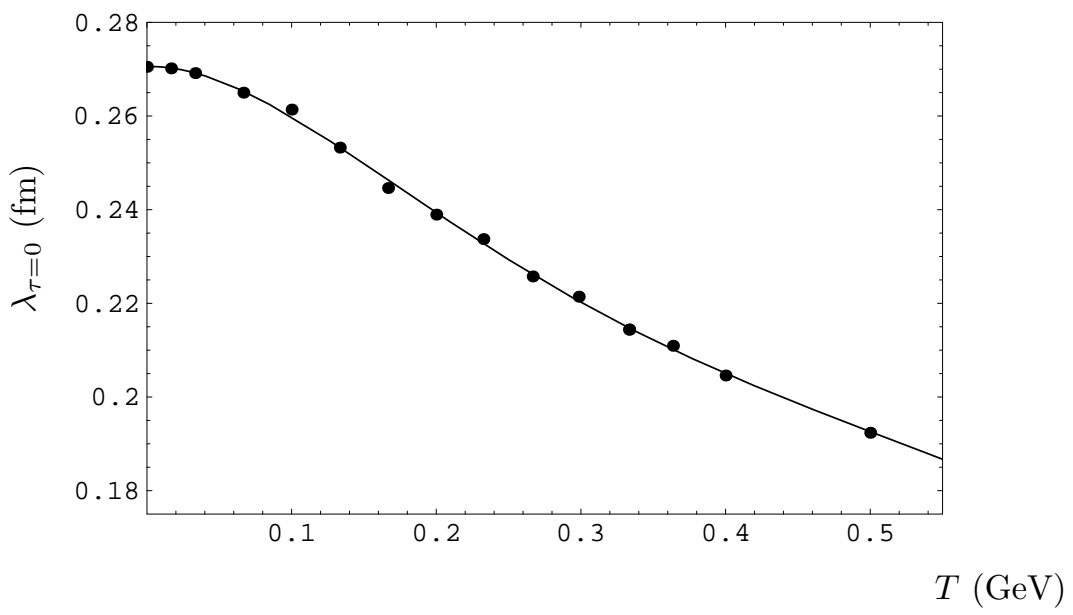

Figure 4: Correlation length as a function of temperature $\lambda_{\tau=0}(T) ; \rho=0.3 \mathrm{fm}$.

this is ruled out by existing lattice calculations, which demonstrate that within errors instanton size distribution stays the same in the temperature region from 0 to $T_{c} 28$.

In this paper we have found the contribution of instanton gas to the bilocal correlator at finite temperature. Comparison of the temperature dependence of correlation length with lattice results allows to make the conclusion that if instanton density is not too high and corrections to the dilute gas approximation are not significant, then instanton density should be much less than $1 \mathrm{fm}^{-4}$, the value standard for instanton liquid model. Vacuum of non-Abelian gauge theory at finite temperature is a very complicated system, and it is natural to expect that it can be a combination of the above listed possibilities.

Authors are grateful to Yu.A. Simonov for useful comments. This work is supported by NSh1774.2003.2 grant and by Federal Program of the Russian Ministry of Industry, Science and Technology No 40.052.1.1.1112. 


\section{References}

[1] E. V. Shuryak, Nucl. Phys. B 203, 93 (1982).

[2] D. Diakonov and V. Y. Petrov, Nucl. Phys. B 245, 259 (1984).

[3] H. G. Dosch, Phys. Lett. B 190, 177 (1987); H. G. Dosch and Y. A. Simonov, Phys. Lett. B 205, 339 (1988); Y. A. Simonov, Nucl. Phys. B 307, 512 (1988).

[4] A. Di Giacomo, H. G. Dosch, V. I. Shevchenko and Y. A. Simonov, Phys. Rept. 372, 319 (2002).

[5] A. I. Shoshi, F. D. Steffen, H. G. Dosch, and H. J. Pirner, Phys. Rev. D 68, 074004 (2003).

[6] M. A. Shifman, A. I. Vainshtein, and V. I. Zakharov, Nucl. Phys. B 163, 46 (1980).

[7] A. B. Migdal, N. O. Agasian and S. B. Khokhlachev, JETP Lett. 41, 497 (1985); N. O. Agasian and S. B. Khokhlachev, Sov. J. Nucl. Phys. 55, 628, 633 (1992).

[8] N. O. Agasian and Y. A. Simonov, Mod. Phys. Lett. A 10, 1755 (1995); N. O. Agasian, Phys. Atom. Nucl. 59, 297 (1996).

[9] N. O. Agasian and S. M. Fedorov, JHEP 0112, 019 (2001); hep-ph/0111305 hep-ph/0211139 Phys. Atom. Nucl. 67, 376 (2004).

[10] G. S. Bali, Phys. Rev. D 62, 114503 (2000).

[11] V. I. Shevchenko and Y. A. Simonov, Phys. Rev. Lett. 85, 1811 (2000); V. I. Shevchenko and Y. A. Simonov, hep-ph/0104135

[12] A. E. Dorokhov, S. V. Esaibegian and S. V. Mikhailov, Phys. Rev. D 56, 4062 (1997).

[13] E. M. Ilgenfritz, B. V. Martemyanov, S. V. Molodtsov, M. Muller-Preussker and Y. A. Simonov, Phys. Rev. D 58, 114508 (1998).

[14] M. D'Elia, A. Di Giacomo and E. Meggiolaro, Phys. Rev. D 67, 114504 (2003).

[15] N. O. Agasian, Phys. Lett. B 562, 257 (2003).

[16] G. Boyd, J. Engels, F. Karsch, E. Laermann, C. Legeland, M. Lutgemeier and B. Petersson, Nucl. Phys. B 469, 419 (1996).

[17] N. A. Campbell, I. H. Jorysz and C. Michael, Phys. Lett. B 167, 91 (1986); I. H. Jorysz and C. Michael, Nucl. Phys. B 302, 448 (1988).

[18] H. G. Dosch, M. Eidemuller and M. Jamin, Phys. Lett. B 452, 379 (1999); M. Eidemuller, H. G. Dosch and M. Jamin, Nucl. Phys. Proc. Suppl. 86, 421 (2000).

[19] Y. A. Simonov, Nucl. Phys. B 592, 350 (2001).

[20] O. Philipsen, Nucl. Phys. B 628, 167 (2002); M. Laine and O. Philipsen, Nucl. Phys. B 523, 267 (1998). 
[21] B. J. Harrington and H. K. Shepard, Phys. Rev. D 17, 2122 (1978).

[22] N. O. Agasian and K. Zarembo, Phys. Rev. D 57, 2475 (1998); N. O. Agasian and D. Antonov, Phys. Lett. B 530, 153 (2002); JHEP 0106, 058 (2001).

[23] T. Schafer and E. V. Shuryak, Rev. Mod. Phys. 70, 323 (1998).

[24] T. C. Kraan and P. van Baal, Phys. Lett. B 435, 389 (1998); K. M. Lee and C. h. Lu, Phys. Rev. D 58, 025011 (1998).

[25] E. M. Ilgenfritz, B. V. Martemyanov, M. Muller-Preussker, S. Shcheredin and A. I. Veselov, Phys. Rev. D 66, 074503 (2002).

[26] E. M. Ilgenfritz, B. V. Martemyanov, M. Muller-Preussker, S. Shcheredin and A. I. Veselov, Nucl. Phys. Proc. Suppl. 119, 754 (2003).

[27] C. Gattringer, E. M. Ilgenfritz, B. V. Martemyanov, M. Muller-Preussker, D. Peschka, R. Pullirsch, S. Schaefer and A. Schafer, Nucl. Phys. Proc. Suppl. 129-130, 653 (2004).

[28] B. Lucini, M. Teper and U. Wenger, arXiv:hep-lat/0401028. 